\title{
Endoscopic Endonasal Transclival Approaches: Case Series and Outcomes for Different Clival Regions
}

\author{
Ryan E. Little ${ }^{1}$ Robert J. Taylor ${ }^{1}$ Justin D. Miller ${ }^{1}$ Emily C. Ambrose ${ }^{1}$ Anand V. Germanwala ${ }^{2}$ \\ Deanna M. Sasaki-Adams ${ }^{3}$ Matthew G. Ewend ${ }^{3}$ Adam M. Zanation ${ }^{1}$
}

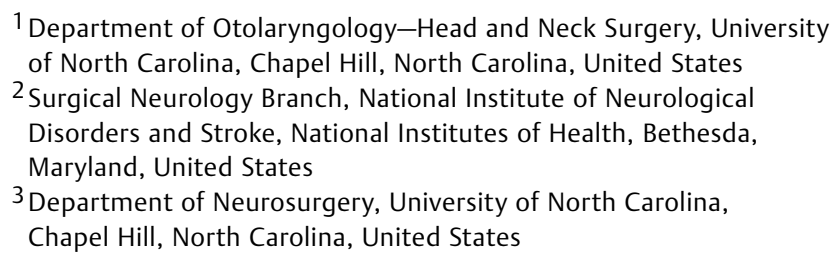

\begin{abstract}
Address for correspondence Adam M. Zanation, MD, Department of Otolaryngology-Head and Neck Surgery, University of North Carolina, Chapel Hill, 170 Manning Drive, Physicians Office Building, Ground Floor, CB\#7070 Chapel Hill, NC 27599, United States (e-mail: adam_zanation@med.unc.edu).
\end{abstract}

J Neurol Surg B 2014;75:247-254.

\begin{abstract}
Keywords

- clivus

- skull base surgery

- endoscopic endonasal approach

- CSF leak

Objective Transclival endoscopic endonasal approaches to the skull base are novel with few published cases. We report our institution's experience with this technique and discuss outcomes according to the clival region involved.

Design Retrospective case series.

Setting Tertiary care academic medical center

Participants All patients who underwent endoscopic endonasal transclival approaches for skull base lesions from 2008 to 2012.

Main Outcome Measures Pathologies encountered, mean intraoperative time, intraoperative complications, gross total resection, intraoperative cerebrospinal fluid (CSF) leak, postoperative CSF leak, postoperative complications, and postoperative clinical course.

Results A total of 49 patients underwent 55 endoscopic endonasal transclival approaches. Pathology included 43 benign and 12 malignant lesions. Mean follow-up was 15.4 months. Mean operative time was 167.9 minutes, with one patient experiencing an intraoperative internal carotid artery injury. Of the 15 cases with intraoperative cerebrospinal fluid (CSF) leaks, 1 developed postoperative CSF leak (6.7\%). There were six other postoperative complications: four systemic complications, one case of meningitis, and one retropharyngeal abscess. Gross total resection was achieved for all malignancies approached with curative intent.

Conclusions This study provides evidence that endoscopic endonasal transclival approaches are a safe and effective strategy for the surgical management of a variety of benign and malignant lesions.

Level of Evidence 4.
\end{abstract}

received

November 27, 2013

accepted

December 15, 2013

published online

April 17, 2014 (c) 2014 Georg Thieme Verlag KG

Stuttgart · New York
DOI http://dx.doi.org/ 10.1055/s-0034-1371522. ISSN 2193-6331. 


\section{Introduction}

The clivus is the unpaired basilar portion of the occipital bone extending obliquely from the anterior aspect of the foramen magnum to join the basisphenoid bone just inferior to the dorsum sellae. Traditionally, lesions of the clivus and surrounding structures have been approached via extended subfrontal transbasal, anterior transfacial, lateral transtemporal, far-lateral approaches, and staged approaches. ${ }^{1-7}$ These approaches are limited in that they often require extensive bone removal and brain retraction while placing critical neurovascular structures between the surgeon and the site of pathology. It has been proposed that these limitations are mitigated with the use of endoscopic endonasal transclival approaches to the skull base. This paradigm shift has been facilitated by advances in rigid endoscopic instrumentation, neuronavigation, and the development of a collaborative model for skull base surgery involving both otolaryngologists and neurosurgeons. Initial outcomes data from small case series for these approaches have been promising, particularly for the treatment of clival chordomas, ${ }^{6-12}$ with several reports describing the feasibility and limitations of the extended endoscopic endonasal transclival approach with respect to regional anatomy. ${ }^{4,9,11,13-15}$

Because so few cases of endoscopic endonasal transclival approaches have been published, the goal of this study is to continue to investigate this surgical technique's safety and efficacy. Clinical outcomes for a diverse group of benign and malignant lesions are presented here based on the clival region involved, and salient cases are discussed to underscore critical regional anatomical considerations and lesion characteristics necessary for surgical planning.

\section{Materials and Methods}

\section{Study Subjects}

This retrospective chart review was approved by the institutional review board at the University of North Carolina at Chapel Hill. All patients undergoing endoscopic endonasal transclival approaches between 2008 and 2012 were identified consecutively from the clinical practice of the senior author. Patient data including demographics, clinical presentation, pathology, mean intraoperative time, intraoperative complications, gross total resection, intraoperative cerebrospinal fluid (CSF) leak, postoperative CSF leak, postoperative complications, and postoperative clinical course were recorded. The follow-up period was defined as the time between surgery to the latest clinical appointment or death.

\section{Surgical Management}

Preoperatively, either computerized tomography or magnetic resonance imaging (MRI) localized the clival lesion and defined the patient's anatomy for surgical planning. Intraoperatively, patient positioning and initial endoscopic endonasal approach to the clivus were performed as was previously described with image guidance utilized in all cases. ${ }^{5,6,13-15}$ Briefly, the neuronavigation system was registered and precision confirmed in three planes. Image guidance was used for the duration of the case. Oxymetazoline-soaked pledgets were placed in bilateral nasal cavities. The patient was prepped and draped in standard fashion. The abdomen was prepped and draped in sterile fashion for cases requiring harvest of a fat graft. Diagnostic bilateral nasal endoscopy was performed at the start of the case using the 0 -degree endoscope. Turbinates were lateralized or harvested for free mucosal graft. A pedicled nasal septal flap was harvested in standard fashion and stored in the nasopharynx. A wide view of the sphenoid rostrum was attained by performing a posterior septectomy and bilateral sphenoidotomies using Kerrison rongeurs and Tru-Cutting instruments. A diamond burr was used to drill the sphenoid rostrum bilaterally and planum sphenoidale. The clivus was drilled as dictated by the lesion characteristics. All cases requiring intradural dissection involved collaboration with a neurosurgeon.

\section{Outcome Assessment by Clival Segments Involved in Approach}

An endoscopic endonasal transclival approach may only involve a segment of the clivus, as required by the location and extension of a particular skull base pathology. Depending on which segment of the clivus is involved in the approach, different neurovascular structures are placed at risk. Thus, to evaluate the outcomes of endoscopic endonasal transclival approaches more specifically, three regions of the clivus were defined. The upper third lies inferior to the dorsum sellae and posterior clinoid processes and superior to the petrous apex, the middle third lies at the level of the petrous segments of the internal carotid artery (ICA), and the inferior third extends from the jugular tubercle to the foramen magnum. ${ }^{16,17}$ Structures at risk for each involved clival segment are shown in -Table 1. Outcomes were examined according to the involved clival segments.

\section{Results}

Forty-nine patients underwent 55 endoscopic endonasal transclival surgeries for 43 benign and 12 malignant lesions (-Table 2). Mean patient age was 49.5 years (standard deviation [SD]: 19.0). Twenty-nine patients were female and 20 were male. Mean operative time was 167.9 minutes with the creation of large skull base defects $(>2 \mathrm{~cm})$ necessary for $85.5 \%$ of procedures. CSF leaks were observed in 15

Table 1 Structures at risk for each involved clival region during expanded endoscopic endonasal transclival approaches

\begin{tabular}{|l|l|l|}
\hline Clival region & Key anatomy & Potential sequelae \\
\hline Superior & $\begin{array}{l}\text { Cavernous } \\
\text { sinus contents }\end{array}$ & $\begin{array}{l}\text { CN III/IV/V1/V2/VI } \\
\text { palsies; intracavernous } \\
\text { carotid artery } \\
\text { hemorrhage }\end{array}$ \\
\hline Middle & $\begin{array}{l}\text { Petrous portion } \\
\text { of the ICA }\end{array}$ & ICA hemorrhage \\
\hline Inferior & Eustachian tube & $\begin{array}{l}\text { Eustachian tube } \\
\text { dysfunction }\end{array}$ \\
\hline
\end{tabular}

Abbreviations: CN, cranial nerve; ICA, internal carotid artery. 
Table 2 Pathology presented in chart review of 49 patients undergoing 55 endoscopic endonasal transclival approaches: 43 benign and 12 malignant lesions

\begin{tabular}{|l|l|l|l|}
\hline Pathology & Cases, $\boldsymbol{n}$ & Pathology & Cases, $\boldsymbol{n}$ \\
\hline Invasive pituitary adenoma & 13 & Hemangiopericytoma & 1 \\
\hline Meningioma & 4 & Lymphoma & 1 \\
\hline Mucocele & 4 & Spindle cell cancer & 1 \\
\hline Invasive fungal sinusitis & 3 & Squamous cell cancer & 1 \\
\hline Chordoma & 3 & Basilar invagination & 1 \\
\hline Metastatic disease & 2 & Encephalocele & 1 \\
\hline Allergic fungal sinusitis & 2 & Clival abscess & 1 \\
\hline Cyst & 2 & Follicular lymphoid hyperplasia & 1 \\
\hline Fibrous dysplasia & 2 & Fungus ball & 1 \\
\hline Foreign body & 2 & Granuloma & 1 \\
\hline Inflammatory mass & 2 & Osteoma & 1 \\
\hline Adenoid cystic carcinoma & 2 & Tornwaldt cyst & 1 \\
\hline Malignant peripheral nerve sheath tumor & 1 & Teratoma & 1 \\
\hline
\end{tabular}

procedures with 10 noted to be high flow. One patient experienced an intraoperative ICA injury. The rate of postoperative to intraoperative CSF leaks was 1 of 15 (6.7\%). Postoperatively, there were four systemic complications, one case of meningitis, and one retropharyngeal abscess. Mean followup was 15.4 months (SD: 13.2). Clinical characteristics and outcomes according to involved clival region are shown in - Table 3.

\section{Superior Clivus}

Nine transclival approaches involved only the superior clivus. Pathology included five invasive pituitary adenomas, one meningioma, one metastatic adenocarcinoma, one inflammatory mass, and one Tornwaldt cyst with cervical spinal extension. Four procedures required intradural dissection.

Intraoperatively, mean surgical time was 146.3 minutes (SD: 34.8). Mean blood loss was $208.3 \mathrm{~mL}$ (SD: 131.1) with no significant neural or vascular intraoperative complications. Postoperatively, mean hospital stay was 2.9 days (SD: 1.5 ). There was one pulmonary embolism on postoperative day 10 . No postoperative infections, CSF leaks, or other systemic complications occurred.

\section{Superior-Middle Clivus}

Eleven transclival approaches involved the superior-middle clivus. Pathology included six invasive pituitary adenomas, one chordoma with extension to sella and planum sphenoidale, one malignant peripheral nerve sheath tumor with extension to petrous apex and infratemporal fossa, one metastatic poorly differentiated squamous cell cancer, one spindle cell cancer, and one allergic fungal sinusitis. Seven procedures required intradural dissection.

Intraoperatively, mean surgical time was 264.9 minutes (SD: 223.2). Mean blood loss was $377.3 \mathrm{~mL}$ (SD: 334.2). Intraoperative injury to the petrous portion of the left ICA occurred with resection of a malignant peripheral nerve sheath tumor. The ICA injury was treated with case cessation and embolization by interventional radiology. The patient experienced transient right gait disturbances that had improved significantly at 6 months. Postoperatively, mean hospital stay was 4.6 days (SD: 5.6 ). One postoperative low-flow CSF leak occurred on postoperative day 4 in the patient with ICA injury. A second patient underwent subsequent planned middle fossa craniotomy for staged resection of residual tumor lateral to the carotid artery. No other postoperative infections or systemic complications occurred.

\section{Middle Clivus}

Twenty-two transclival approaches involved the middle clivus. Pathology included three meningiomas, two invasive pituitary adenomas, two adenoid cystic carcinomas, two fibrous dysplasia with extension to orbit, two mucoceles, two foreign bodies, one hemangiopericytoma, one granuloma, one osteoma with extension to bilateral ICA, one teratoma, one encephalocele, one invasive fungal sinusitis, one fungus ball with extension to the petrous apex, orbit, and cavernous sinus, one allergic fungal sinusitis, and one inflammatory mass. Three procedures required intradural dissection.

Intraoperatively, mean surgical time was 125.3 minutes (SD: 65.5). Mean blood loss was $125.2 \mathrm{~mL}$ (SD: 103.0) with no significant neural or vascular intraoperative complications. Postoperatively, mean hospital stay was 7.5 days (SD: 20.5). One patient remained intubated following resection of invasive fungal sinusitis, developed recurrent aspiration pneumonias requiring tracheostomy and gastrostomy tube placement, and was later noted to have a subacute infarct during a subsequent hospital admission. Another patient was readmitted within 30 days for acute renal failure due to dehydration and amphotericin renal toxicity. No other postoperative infections, systemic complications, or CSF leaks occurred. 


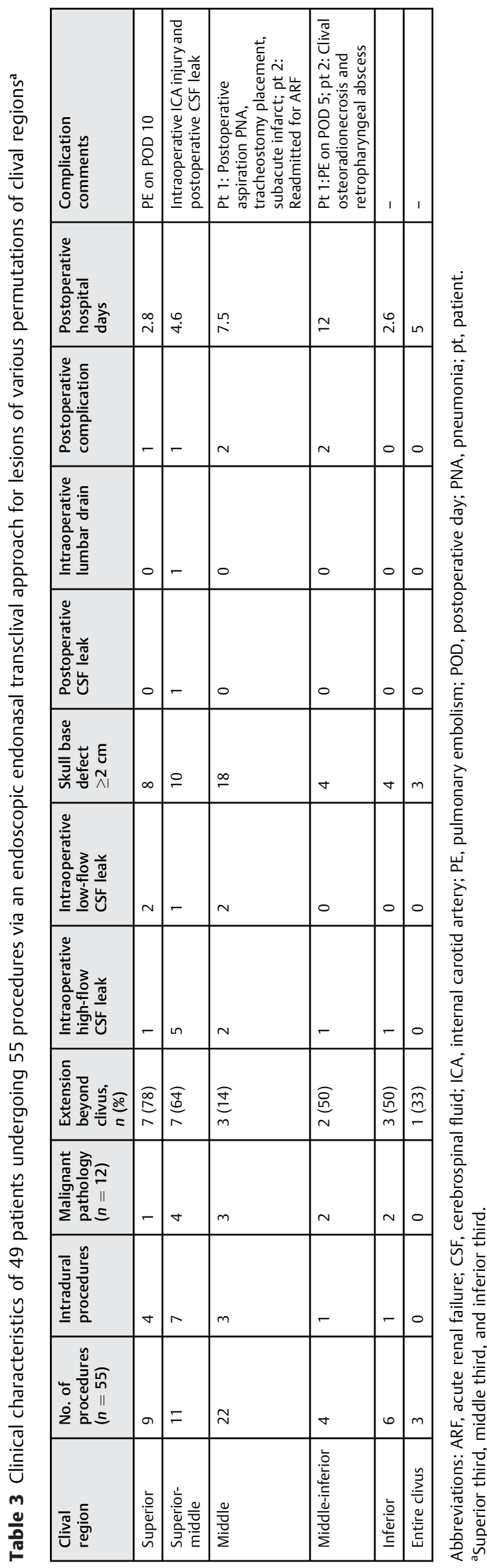

\section{Middle-Inferior Clivus}

Four transclival approaches involved the middle-inferior clivus. Pathology included a two-stage chordoma operation notable for extension to the spine and two mucoceles. One procedure required intradural dissection. One patient with a remote history of clival chordoma resection via transpalatal approach with adjuvant radiation therapy underwent a transclival approach for a mucocele. One year later the patient developed a retropharyngeal abscess and was noted to have clival osteoradionecrosis requiring complete resection of the clivus with an adipofascial radial forearm flap.

Intraoperatively, mean surgical time was 256 minutes (SD: 227.8). Mean blood loss was $325 \mathrm{~mL}$ (SD 206.2) with no significant neural or vascular intraoperative complications. Postoperatively, mean hospital stay was 12 days (SD: 7.8). There was one pulmonary embolism on postoperative day 5 . No other postoperative infections, CSF leaks, or systemic complications occurred.

\section{Inferior Clivus}

Six transclival approaches involved the inferior clivus. Pathology included one squamous cell cancer, one lymphoma with extension to nasopharynx, one basilar invagination with extension to spine, two cysts, and one follicular lymphoid hyperplasia. One cyst required combined endoscopic endonasal transclival and transoropharyngeal approaches for extension to the cervical spine. One procedure required intradural dissection.

Intraoperatively, mean surgical time was 91.8 minutes (SD: 65.9). Mean blood loss was $266.7 \mathrm{~mL}$ (SD: 230.7) with no significant neural or vascular intraoperative complications. Postoperatively, mean hospital stay was 2.3 days (SD: 3.3). No postoperative infections, systemic complications, or CSF leaks occurred.

\section{Entire Clivus}

Three transclival approaches involved the entire clivus. Pathology included two invasive fungal sinusitis resections and one clival abscess. No procedures required intradural dissection.

Intraoperatively, mean surgical time was 223.3 minutes (SD: 94.1). Mean blood loss was $128.3 \mathrm{~mL}$ (SD: 202.4) with no significant neural or vascular intraoperative complications. Postoperatively, mean hospital stay was 5 days (SD: 3 ). No postoperative infections, systemic complications, or CSF leaks occurred.

\section{Malignant Conditions}

Eleven patients underwent endoscopic endonasal transclival approaches for skull base malignancies. Clinical characteristics and outcomes for these patients are shown in - Table 4 . Mean preoperative MRI size was $3.8 \mathrm{~cm}$ (SD: 1.3). Gross total resection (GTR) was achieved in all cases where curative intent was the goal. Mean follow-up for patients undergoing surgical resection for curative intent was 19.7 months (SD: 12.3). Four of these patients had no evidence of disease recurrence at the time of this study. One patient developed local recurrence of adenoid cystic carcinoma at 18 months. 


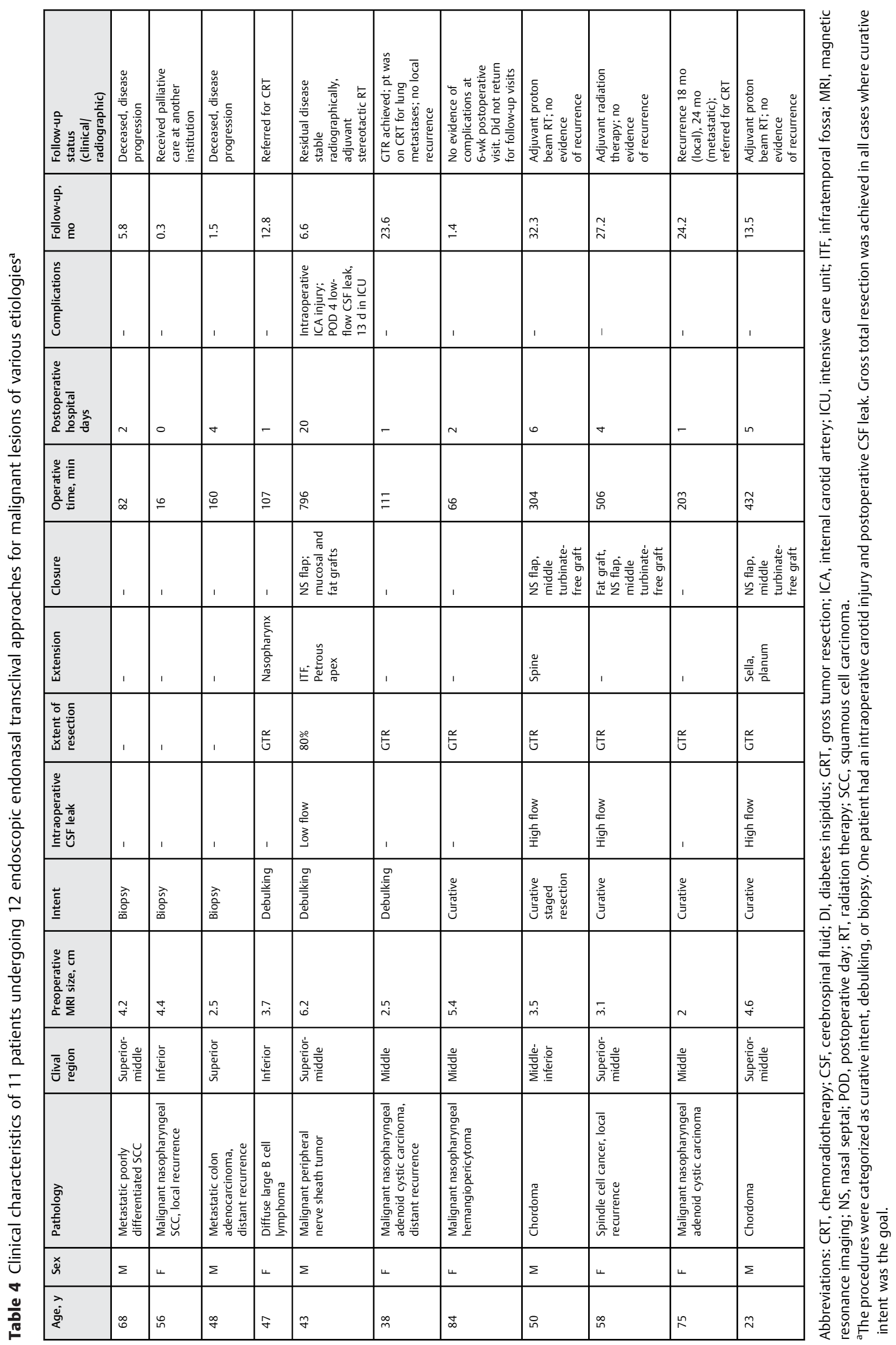


Adjuvant proton radiation therapy was utilized for both clival chordomas, and there was no evidence of disease at 13.5 and 32.3 months.

\section{Discussion}

This retrospective chart review of 49 patients who underwent endoscopic endonasal transclival approaches for a variety of skull base lesions is one of only a few studies demonstrating outcomes by clival region involved. Additionally, there are limited data regarding the use of this approach for benign and malignant intradural lesions. This study provides much needed clinical data for this increasingly utilized approach. The long-term clinical outcomes presented in this case series provide further support for the safety and efficacy of expanded endoscopic endonasal transclival approaches.

Extended endoscopic endonasal approaches to the clivus have been increasingly described by several groups, particularly as an option for the surgical management of malignancies. $^{4-10,13-15}$ Our postoperative to intraoperative CSF leak rate was $6.7 \%$ for all cases and $25 \%$ among malignancies. This rate is similar to previously reported rates of up to $33 \%$ for clival chordomas. ${ }^{6-8,10,19,20}$ GTR was achieved in $100 \%$ of malignant cases approached with curative intent. This included two clival chordomas. Prior case series of the endoscopic endonasal transclival approach to clival chordomas report a GTR rate of 55 to $83 \%{ }^{6-8}$ Additionally, the high rates of disease-free survival demonstrated in this study provide further support for the use of these approaches for the surgical management of malignant lesions.

Important anatomical considerations for the expanded endoscopic endonasal approach to various clival regions has been previously described. ${ }^{14,15,18}$ There was 1 intraoperative ICA injury out of 55 procedures, which was consistent with previously reported rates of up to $2 \%{ }^{10}$ This 43 year-old woman with a history of neurofibromatosis type 1 reported a remote history of craniotomy with radiation therapy for a right-sided brain tumor. Medical records were
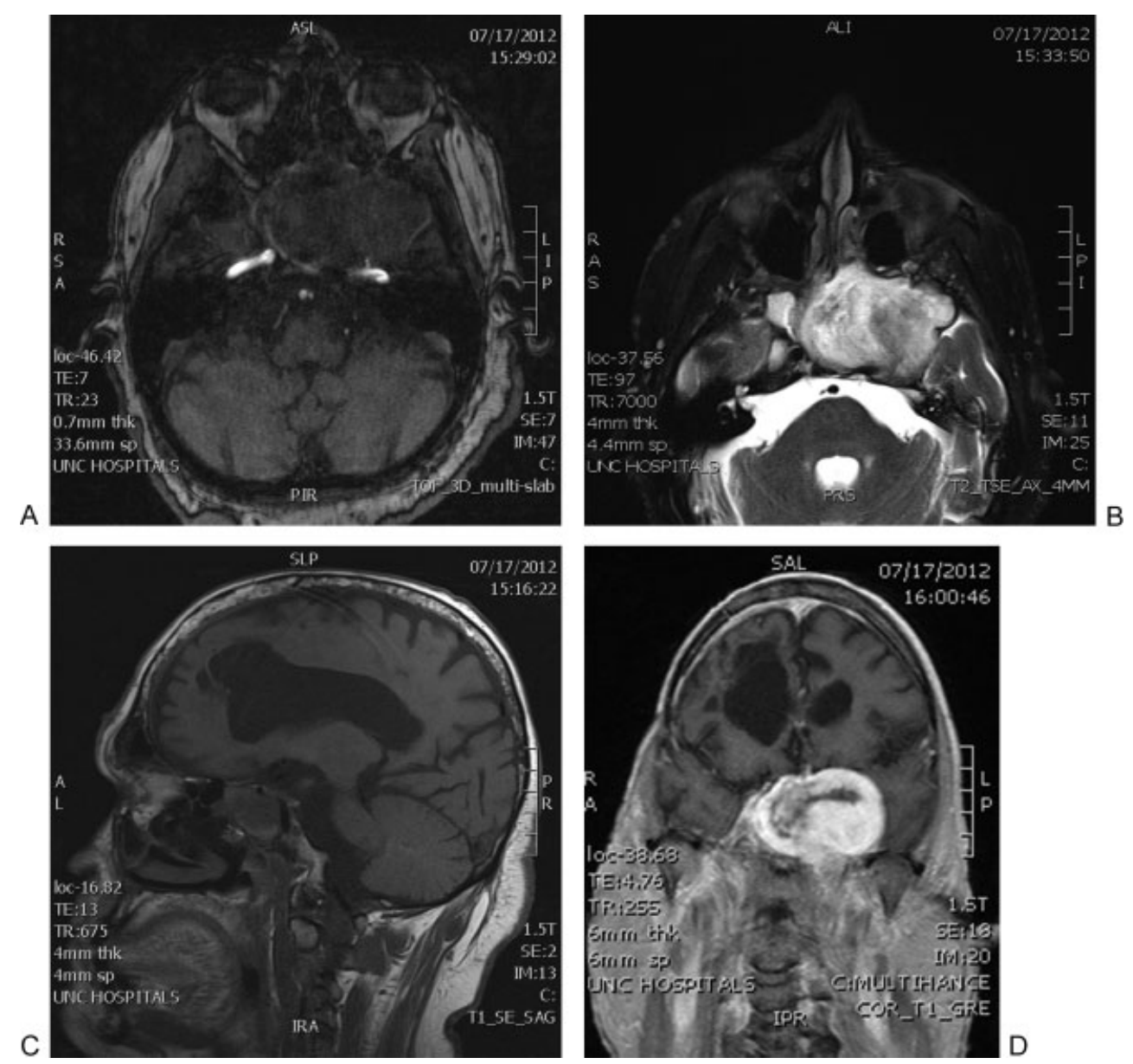

Fig. 1 A 43-year-old woman underwent an endoscopic endonasal transclival approach to a $6.2 \mathrm{~cm} \times 4.0 \mathrm{~cm}$ left malignant peripheral nerve sheath tumor. The patient presented with a 2-year history of headaches and progressive left-sided vision loss. Preoperative magnetic resonance imaging (MRI) and angiogram revealed tumor extension to the petrous apex and infratemporal fossa with posterosuperior displacement of the petrous and proximal cavernous portions of the left internal carotid artery (ICA) with $45 \%$ narrowing at these segments. Magnetic resonance angiography (MRA) also revealed tumor blush arising directly from the ICA. Intraoperative injury to the petrous portion of the left ICA was treated with case cessation and embolization by interventional radiology. During subsequent attempted clip ligation, tumor invasion into the ICA was noted. MRI demonstrated extension of lesion and relationship to left ICA. (A) Axial three-dimensional time-of-flight MRA. (B) Axial T2. (C) Sagittal T1. (D) Coronal T1 sections. 
no longer available. The patient presented to our institution with a 2-year history of headaches and progressive left-sided vision loss. Outside imaging demonstrated a large heterogeneously enhancing mass in the region of the left cavernous sinus. Endoscopic endonasal biopsy showed a grade 2 malignant peripheral nerve sheath tumor. Preoperative MRI and angiogram demonstrated a $6.2-\mathrm{cm}$ lesion extending to the petrous apex and infratemporal fossa with posterior and superior displacement of the petrous and proximal cavernous portions of the left ICA ( - Fig. 1). Tumor blush was identified from small arteries arising from the petrous segment of the ICA. Intraoperatively, Doppler probe was used to identify the left parapharyngeal carotid artery as well as the artery posterior to the tumor in the left clival area. Arterial injury at the level of the left carotid second genu occurred during mobilization of the tumor from the artery. Invasion of the carotid artery by the tumor and retrograde blood flow was noted with attempted clip ligation of the proximal cavernous portion of the ICA. In this case, expanded endoscopic endonasal tumor resection was complicated by vascular invasion. A CSF leak was observed on postoperative day 4. This lowflow leak, which was separate from the intact nasal septal flap, was managed with mucosal and fat grafts. Although uncommon, intraoperative ICA injury during endoscopic endonasal transclival approaches has been previously reported as a major limitation to using this approach. Important considerations for the safe and effective use of this approach include size and lateral extension of the lesion relative to the ICA, preoperative surgical intent, and an experienced surgical and interventional radiology team. ${ }^{6}$

This study further supports the safety and efficacy of the endoscopic endonasal transclival approach for a large cohort of patients and stratifies clinical data according to clival region involved. There are limited data regarding the use of this approach for intradural and extradural lesions, and this study provides much needed clinical data for this increasingly utilized approach. In this study, the overall risk of complications was low and comparable with other reports. ${ }^{5-8,10}$ Additionally, the high rates of GTR and the long-term outcomes of this approach, when combined with appropriate adjuvant therapies, demonstrated encouraging rates of progression free-survival and local tumor control.

\section{Study Limitations}

Given the retrospective nature of these data, there are several limitations to our study. The small number of malignant conditions limits the statistical power of any conclusions that one may draw from this report. Additionally, the conditions treated by this approach represent a diverse group of pathologies referred to a tertiary care academic medical center with variable degrees of complexity and patient comorbidities. Finally, this review of a single surgeon's operative experience may limit the reproducibility and generalizability of these outcomes.

\section{Conclusion}

This study provides evidence that endoscopic endonasal transclival approaches to the clivus are a safe and effective strategy for the surgical management of benign and malignant lesions with a low risk of complications for carefully selected patients.

\section{Acknowledgments}

This work was supported by a grant from the Doris Duke Charitable Foundation to the University of North Carolina at Chapel Hill to fund Clinical Research Fellow Robert Taylor.

\section{Conflict of Interest}

The authors have nothing to disclose.

\section{References}

1 Solares CA, Fakhri S, Batra PS, Lee J, Lanza DC. Transnasal endoscopic resection of lesions of the clivus: a preliminary report. Laryngoscope 2005;115(11):1917-1922

2 Mortini P, Roberti F, Kalavakonda C, Nadel A, Sekhar LN. Endoscopic and microscopic extended subfrontal approach to the clivus: a comparative anatomical study. Skull Base 2003;13(3): 139-147

3 Crumley RL, Gutin PH. Surgical access for clivus chordoma. The University of California, San Francisco, experience. Arch Otolaryngol Head Neck Surg 1989;115(3):295-300

4 Carrabba G, Dehdashti AR, Gentili F. Surgery for clival lesions: open resection versus the expanded endoscopic endonasal approach. Neurosurg Focus 2008;25(6):E7

5 Fraser JF, Nyquist GG, Moore N, Anand VK, Schwartz TH. Endoscopic endonasal minimal access approach to the clivus: case series and technical nuances. Neurosurgery 2010;67(3, Suppl Operative):ons150-ons158; discussion ons158

6 Raper DMS, Komotar RJ, Fraser JF, Anand VK, Moore N, Schwartz TH. Skull base chordomas: endonasal endoscopic transclival approach. In: Hayat M, ed. Astrocytoma, Medulloblastoma, Retinoblastoma, Chordoma, Craniopharyngioma, Oligodendroglioma, and Ependymoma. New York, NY: Springer; 2012:185-194. Tumors of the Central Nervous System; vol 8

7 Fraser JF, Nyquist GG, Moore N, Anand VK, Schwartz TH. Endoscopic endonasal transclival resection of chordomas: operative technique, clinical outcome, and review of the literature. J Neurosurg 2010;112(5):1061-1069

8 Koutourousiou M, Gardner PA, Tormenti MJ, et al. Endoscopic endonasal approach for resection of cranial base chordomas: outcomes and learning curve. Neurosurgery 2012;71(3): 614-624; discussion 624-625

9 Hong Jiang W, Ping Zhao S, Hai Xie Z, Zhang H, Zhang J, Yun Xiao J. Endoscopic resection of chordomas in different clival regions. Acta Otolaryngol 2009;129(1):71-83

10 Saito K, Toda M, Tomita T, Ogawa K, Yoshida K. Surgical results of an endoscopic endonasal approach for clival chordomas. Acta Neurochir (Wien) 2012;154(5):879-886

11 Cavallo LM, Cappabianca P, Messina A, et al. The extended endoscopic endonasal approach to the clivus and cranio-vertebral junction: anatomical study. Childs Nerv Syst 2007;23(6):665-671

12 Frank G, Sciarretta V, Calbucci F, Farneti G, Mazzatenta D, Pasquini E. The endoscopic transnasal transsphenoidal approach for the treatment of cranial base chordomas and chondrosarcomas. Neurosurgery 2006;59(1, Suppl 1):ONS50-ONS57; discussion ONS50-ONS57

13 Kassam AB, Gardner P, Snyderman CH, Mintz A, Carrau RL. Expanded endonasal approach: fully endoscopic, completely 
transnasal approach to the middle third of the clivus, petrous bone, middle cranial fossa, and infratemporal fossa. Neurosurg Focus 2005;19(1):E6

14 Kassam A, Snyderman CH, Mintz A, Gardner P, Carrau RL. Expanded endonasal approach: the rostrocaudal axis. Part I. Crista galli to the sella turcica. Neurosurg Focus 2005;19(1):E3

15 Kassam A, Snyderman CH, Mintz A, Gardner P, Carrau RL. Expanded endonasal approach: the rostrocaudal axis. Part II. Posterior clinoids to the foramen magnum. Neurosurg Focus 2005;19(1):E4

16 Burkart CM, Theodosopoulos PV, Keller JT, Zimmer LA. Endoscopic transnasal approach to the clivus: a radiographic anatomical study. Laryngoscope 2009;119(9):1672-1678
17 Goravalingappa R, Han JC, Mangiardi J, Levenson M. Endoscopic/ Microscopic approach to sphenopetroclival complex: an anatomical study. Skull Base Surg 1999;9(1):33-39

18 Kassam AB, Vescan AD, Carrau RL, et al. Expanded endonasal approach: vidian canal as a landmark to the petrous internal carotid artery. J Neurosurg 2008;108(1):177-183

19 Singh A, Germanwala AV. Management of postoperative complications of skull base surgery. Oper Tech Otolaryngol Head Neck Surg 2011;22(3):237-245

20 Liu JK, Schmidt RF, Choudhry OJ, Shukla PA, Eloy JA. Surgical nuances for nasoseptal flap reconstruction of cranial base defects with high-flow cerebrospinal fluid leaks after endoscopic skull base surgery. Neurosurg Focus 2012;32(6):E7 